\title{
Associations between readmission and patient-reported measures in acute psychiatric inpatients: a study protocol for a multicenter prospective longitudinal study (the ePOP-J study)
}

Sosei Yamaguchi ${ }^{1 *} \mathbb{0}$, Yasutaka Ojio ${ }^{1}$, Junko Koike ${ }^{1}$, Asami Matsunaga' ${ }^{1}$, Makoto Ogawa ${ }^{1}$, Hisateru Tachimori ${ }^{2,3}$, Akiko Kikuchi ${ }^{1}$, Hiroshi Kimura ${ }^{4,5}$, Ataru Inagaki ${ }^{6}$, Hiroyuki Watanabe ${ }^{5,7}$, Yoshiki Kishi ${ }^{8}$, Koji Yoshida ${ }^{9}$, Takaaki Hirooka ${ }^{10}$, Satoru Oishi ${ }^{10}$, Yasuhiro Matsuda ${ }^{11}$ and Chiyo Fujii ${ }^{1}$

\begin{abstract}
Background: Several previous observational studies have reported the risk factors associated with readmission in people with mental illness. While patient-reported experiences and outcomes have become increasingly important in healthcare, only a few studies have examined these parameters in terms of their direct association with readmission in an acute psychiatric setting. This project will investigate multiple factors associated with readmission and community living in acute psychiatric patients in Japan. This study will primarily investigate whether patient-reported experiences at discharge, particularly quality of life (QoL), are associated with future readmission and whether readmission after the index hospitalization is associated with changes in patient-reported outcomes during the study period. Here, we describe the rationale and methods of this study.

Methods: This multicenter prospective cohort study is being conducted in 21 participating Japanese hospitals, with a target sample of approximately 600 participants admitted to the acute psychiatric ward. The study has four planned assessment points: time of index admission (T1), time of discharge (from the index admission) (T2), 6 months after discharge from the index admission (T3), and 12 months after discharge from the index admission (T4). Participants will complete self-reported measures including a QoL scale, a subjective disability scale, and an empowerment- and self-agency-related scale at each assessment point; additionally, service satisfaction, subjective view of need for services, and subjective relationships with family members will be assessed at T2 and T3. We will assess the participants' hospitalization during the study period and evaluate several potential individual- and service-level factors associated with readmission and patient-reported experiences and outcomes. Multivariate analyses will be conducted to identify potential associations between readmission and patient-reported experiences and outcomes.
\end{abstract}

Discussion: The present study may produce evidence on how patient-reported experiences at discharge influence readmission and on the influence of readmission on the course of patient-reported outcomes from admission to community living after discharge. The study may contribute to improving care for both patients'subjective views of their own health conditions and their community lives in an acute psychiatric setting.

\footnotetext{
*Correspondence: sosei.yama@ncnp.go.jp

${ }^{1}$ Department of Community Mental Health \& Law, National Institute of Mental Health, National Center of Neurology and Psychiatry, 4-1-1

Ogawa-Higashi, Kodaira 187-8553, Japan

Full list of author information is available at the end of the article
} 
Trial registration University Hospital Medical Information Network—Clinical Trials Registry (UMIN-CTR) UMIN000034220. Registered on September 20, 2018.

Keywords: Longitudinal study, Patient-reported experience, Patient-reported outcome, Psychiatric hospital, Readmission

\section{Background}

Over the past 50 years, there have been two predominant themes in psychiatric care worldwide, namely, community living and personal recovery for people with mental illness, which focus on the individual processes through which people with mental illness subjectively achieve meaningful lives $[1,2]$. With regard to community living, as the number of patients who live in the community has increased through deinstitutionalization in developed countries [3-5], several studies have examined factors associated with readmission, which has a negative impact on sustainable community living for people with mental illness [6-16]. Previous relevant studies and systematic reviews have confirmed several individual- and service-level factors associated with readmission [6-16]. Individual-level factors include diagnosis, sex, age, treatment adherence, symptoms, housing problems, problematic behaviors, past hospitalization, and comorbidities. Service-level factors include inpatient care programs, medications, provision of care after discharge, other types of comprehensive community care and locations of hospitals and patients [11-16]. In addition, a recent study that sought to identify the various risks for readmission developed and validated a comprehensive index called the READMIT, whose score was associated with future readmission among psychiatric inpatients [17].

While several objective factors at patient discharge have been reported to be risks for future readmission, patients' subjective experiences may also be associated with readmission. If patients' subjective views of themselves at discharge or their experiences during hospitalization can be shown to be independent risk factors for their future readmission, psychiatric care should focus on improving these factors. Indeed, patient-reported assessments of QoL and personal recovery have been somewhat correlated with symptoms and functions $[18,19]$. In addition, one recent study reported a potential association between subjective QoL and future readmission among patients with schizophrenia in Israel [20]. However, a systematic review also noted that few studies have examined the association between subjective patient views and readmission [11]. Therefore, the relationship between these variables is still hypothetical.

As another example of subjective experience and readmission, psychiatric patients may experience traumatic treatment during their hospitalization, particularly in an acute care or compulsory treatment settings [21, 22]. Poor service engagement caused by negative perceptions of the treatment received is likely to adversely affect clinical outcomes [23]. Indeed, in a large-scale longitudinal study, satisfaction with inpatient care was associated with readmission in acute inpatients with schizophrenia [24]. However, a systematic review reported inconsistent findings regarding the associations between patients' views of care quality and prognostic outcomes, including readmission [22]. In short, there is still limited evidence on the relationship between patient-reported treatment experiences and readmission.

In parallel with the personal recovery movement worldwide, the interest in patient-reported outcomes has dramatically increased in psychiatric care $[19,25-$ $28]$. Conceptually, the personal recovery process does not end even when a patient faces a crisis linked to readmission [29]. However, a small number of studies have examined whether readmission affects changes in patient-reported outcomes or reported the potential factors that affect patient-reported outcomes after hospital discharge. For example, one German study did not find any variables that directly affected changes in QoL scores between admission and 9-month follow-up in schizophrenia inpatients, but the study suggested that depressive symptoms were a potential factor [30]. Another German randomized control trial of a psychoeducation and monitoring program reported the potential influence of compulsory rehospitalization on changes in self-reported mental health functioning during a 2-year follow-up [31]. In addition, past studies in inpatient and outpatient settings identified factors potentially associated with changes in QoL or empowerment, including age, male sex, diagnosis (e.g., schizophrenia and bipolar disorders), and length of hospital stay [31-33], although patient-reported outcomes, particularly QoL, are likely to vary among post discharge residential service settings, such as outreach services $[34,35]$. In sum, while past studies have identified potential factors that influence the prognostic value of patient-reported outcomes, there is still insufficient evidence on the direct association between changes in patient-reported outcomes (e.g., QoL, empowerment, and self-reported functional impairment) and readmission, particularly among patients in the acute phase. 
In terms of the Japanese context, Japan is undergoing a transition from inpatient care to community mental health care. Most psychiatric care is still provided in inpatient settings [36]; the average length of psychiatric hospitalization is approximately 300 days [37]. The Japanese government has reformed relevant laws to prevent long-term inpatient care starting in the 21st century. Clinicians have started to develop systems for community care $[37,38]$. These changes have resulted in the fact that more than $80 \%$ of inpatients were discharged within 1 year [39]. Nevertheless, approximately $40 \%$ of inpatients are rehospitalized within 1 year after discharge [38]. Some recent studies in Japan based on analyses of the national database have noted that highly intensive medical support delivered as part of inpatient care and followup visits has potentially reduced the risk of readmission $[40,41]$. However, most Japanese empirical studies on psychiatric readmission have focused on the system level. Few studies have comprehensively examined either the individual factors that predict readmission after discharge or patient-reported outcomes after discharge, particularly in acute psychiatric care settings. In other words, both clinical variables and patient-reported measures related to hospitalization and community living in Japan remain unclear.

\section{Aims and objectives}

To address the evidence gap, we initiated the ePOP-J study (early discharge and Prognostic community Outcomes for Psychiatric inpatients in Japan: a longitudinal study), which is a multicenter prospective cohort study designed to investigate multiple factors, including patient-reported measures, associated with readmission and community living, in acute psychiatric patients in Japan. The overarching aim of the present study is to explore the association between patient-reported experiences and outcomes and the incidence of readmission during 12 months of follow-up. In this study, patientreported measures, particularly QoL, will be used to assess both exposures and outcomes. The study primarily aims to evaluate the following associations and factors, although we will also perform a comprehensive exploratory analysis to identify other (non-patient-reported) factors for readmission after evaluating the described associations and factors:

1. The relationship between readmission during the 12-month follow-up beginning at discharge after the index admission (primary outcome), and the QoL score at discharge (primary exposure).

2. The association between readmission during the 12-month follow-up beginning at discharge after the index admission and the self-reported disability score, empowerment/self-agency-related measure score or inpatient care service satisfaction score at discharge.

3. Influence of readmission on changes in scores for patient-reported outcome measures (QoL, selfreported disability and empowerment/self-agencyrelated outcome) during the study period.

4. Other potential factors influencing score changes in patient-reported outcome measures, such as age, sex, diagnosis, length of hospital stay, living conditions, and utilization of outreach services after discharge.

\section{Methods and analysis}

\section{Study design and settings}

A multicenter prospective cohort study has been planned. The study protocol was designed by professional researchers, clinicians, and mental health services users. Specifically, our project team has involved a mental health service user researcher as a core member, and we collaboratively determined the study participants' eligibility criteria, recruitment methods, and outcomes and exposures. We employed a prospective design for this study to repeatedly assesses patient-reported experiences and outcomes in situations where these measures are not routinely evaluated in the current mental health care system in Japan. We recruited 21 psychiatric hospitals with acute wards. Because human resources that perform research work vary considerably among psychiatric hospitals in Japan, we needed to recruit only hospitals with sufficient staff members to conduct a prospective study. Therefore, we used a snowball sampling method across Japan instead of using random sampling. The study will assess acute ward inpatients' exposures and outcomes at admission (T1), discharge (T2), 6 months after discharge from the index admission (T3), and 12 months after discharge from the index admission (T4). We will also evaluate exposures and outcomes among inpatients who cannot be discharged within 12 months of the index admission (Fig. 1). Patient recruitment began on 1 October 2018.

\section{Eligibility criteria Inclusion criteria}

Eligible participants are patients diagnosed with psychiatric disorders based on the International Classification of Diseases 10th revision (F00-F90) who require treatment in the acute psychiatric ward of participating psychiatric hospitals between 1 October 2018 and 30 September 2019. The main analysis will include only participants discharged from each participating hospital within 12 months. 


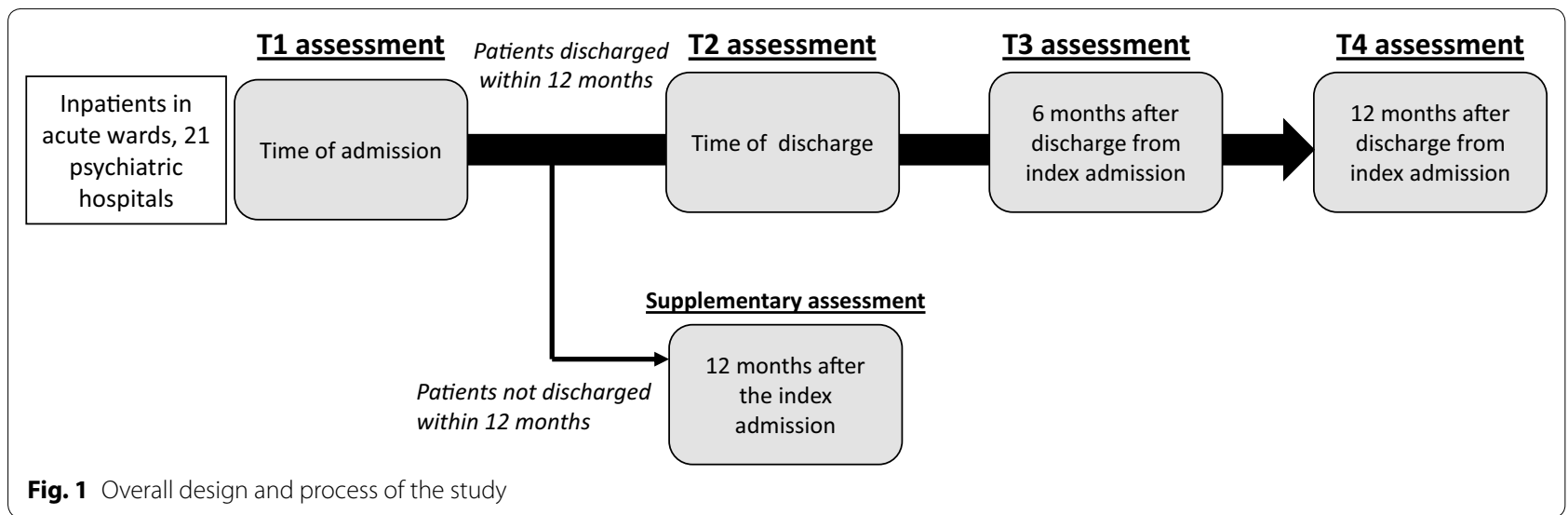

\section{Exclusion criteria}

Exclusion criteria are as follows: age younger than 20 years or older than 60 years and hospitalization for specific tests and treatments (e.g., psychiatric testimony, electroconvulsive therapy). Patients are also excluded if they are immediately moved to other medical departments for long-term care after hospitalization (e.g., if a severe physical disorder was found) or if they receive psychiatric treatment outside of participating hospitals or their affiliated medical institutions.

\section{Procedures for informed consent}

When a potential participant is admitted to the acute ward of a participating psychiatric hospital, staff members, including psychiatrists, nurses, occupational therapists, and social workers, screen the patient against the eligibility criteria. If an inpatient fulfils all the criteria and has the cognitive capacity for informed consent, trained staff members will provide a description of the study and the ethical issues involved using the descriptive brochure approved by the ethics committee. Informed consent will be obtained from the participant as soon as possible, within approximately 2 weeks after admission. Each hospital will recruit a maximum of 10 participants per month.

\section{Procedures for data collection}

After informed consent is obtained, participants will complete a self-administered questionnaire at the time of admission (T1). The staff members will assist by reading the questionnaire if necessary. Trained staff members will also complete the first assessment sheet, which includes sociodemographic information and past medical history, based on medical records. In addition, they will assess staff-rated measures based on observation and a brief interview with the participant. At discharge (T2), the participant will complete the self-administered questionnaire again. Next, staff members will complete staff-rated measures and will document the types of inpatient care that were provided during hospitalization on the second assessment sheet, based on medical records. At 6 months (T3) and 12 months (T4) after the index admission, the participant will complete a self-administered questionnaire, and staff members will complete an assessment sheet detailing staff-rated measures, readmission, and outpatient services. To prevent loss to follow-up at 6 months (T3) and 12 months (T4), staff members in the inpatient care department, outpatient care department, and outreach team will cooperate to share information on each participant's status.

For participants who are not discharged within 12 months of the index admission, the same procedure used for the discharge survey (T2) will be conducted when the hospital stay reaches 365 days. At this time, the patient's survey will be concluded. The main analysis will only include participants who are discharged within 12 months.

Data monitoring and management will be conducted by the Department of Community Mental Health and Law, National Center of Neurology and Psychiatry, which is independent from the grant funder. At least two researchers or research assistants will check the data to maintain data quality.

\section{Research measures and valuables}

The variables assessed at each time point in this study are summarized in Table 1.

\section{Patient-reported measures}

The study assesses three patient-reported experience and outcome measures at all time points. The EuroQOL Five Dimensions and Five Levels (EQ-5D-5L) will be used as the primary measure of interest (primary exposure) 
Table 1 Collection of data on exposures, outcomes, and other explanatory variables at each assessment point

\begin{tabular}{|c|c|c|c|c|}
\hline Item & $\begin{array}{l}\text { Time } \\
\text { of admission } \\
\text { (T1) }\end{array}$ & $\begin{array}{l}\text { Time } \\
\text { of discharge } \\
\text { (T2) }\end{array}$ & $\begin{array}{l}\text { 6-month } \\
\text { follow-up } \\
\text { (T3) }\end{array}$ & $\begin{array}{l}\text { 12-month } \\
\text { follow-up } \\
\text { (T4) }\end{array}$ \\
\hline \multicolumn{5}{|l|}{ Patient-reported outcomes/experiences } \\
\hline Quality of life (EQ-5D-5L) & $\checkmark$ & $\checkmark$ & $\checkmark$ & $\checkmark$ \\
\hline Perception of disabilities (SDS-J) & $\checkmark$ & $\checkmark$ & $\checkmark$ & $\checkmark$ \\
\hline Empowerment and self-agency related scale & $\checkmark$ & $\checkmark$ & $\checkmark$ & $\checkmark$ \\
\hline Satisfaction with care & & $\checkmark$ & $\checkmark$ & \\
\hline Subjective relationship with family & & $\checkmark$ & $\checkmark$ & \\
\hline \multicolumn{5}{|l|}{ Clinical and social variables } \\
\hline \multicolumn{5}{|l|}{ Past hospitalisation or readmission } \\
\hline Number of admissions ${ }^{a}$ & $\checkmark$ & $\checkmark$ & $\checkmark$ & $\checkmark$ \\
\hline Length of hospitalisation ${ }^{\mathrm{a}}$ & $\checkmark$ & $\checkmark$ & $\checkmark$ & $\checkmark$ \\
\hline Type of hospitalisation (voluntary/involuntary)a & $\checkmark$ & $\checkmark$ & $\checkmark$ & $\checkmark$ \\
\hline Reasons for emergency admission ${ }^{\mathrm{a}}$ & $\checkmark$ & $\checkmark$ & $\checkmark$ & $\checkmark$ \\
\hline Unplanned discharge from index admission ${ }^{a}$ & & $\checkmark$ & & \\
\hline Psychiatric present status ${ }^{\mathrm{b}, \mathrm{c}}$ & $\checkmark$ & & & \\
\hline Symptoms (1 item) & $\checkmark$ & $\checkmark$ & $\checkmark$ & $\checkmark$ \\
\hline Social relationships (1 item) & $\checkmark$ & & $\checkmark$ & $\checkmark$ \\
\hline Employment status & $\checkmark$ & $\checkmark$ & $\checkmark$ & $\checkmark$ \\
\hline Functioning (PSP) & $\checkmark$ & $\checkmark$ & $\checkmark$ & $\checkmark$ \\
\hline History of problematic behaviours ${ }^{b}$ & $\checkmark$ & $\checkmark$ & $\checkmark$ & $\checkmark$ \\
\hline Risk of problematic behaviours & $\checkmark$ & $\checkmark$ & $\checkmark$ & $\checkmark$ \\
\hline Weight & $\checkmark$ & $\checkmark$ & $\checkmark$ & $\checkmark$ \\
\hline Body mass index & $\checkmark$ & $\checkmark$ & $\checkmark$ & $\checkmark$ \\
\hline \multicolumn{5}{|l|}{ Other exposure variables } \\
\hline \multicolumn{5}{|l|}{ Socio-demographics } \\
\hline Age $^{a}$, sex, living condition, living location, social security etc. & $\checkmark$ & & & \\
\hline \multicolumn{5}{|l|}{ Diagnostic information ${ }^{\mathrm{a}}$} \\
\hline Psychiatric disorders ${ }^{\mathrm{a}}$ & $\checkmark$ & $\checkmark$ & & \\
\hline Physical disorders ${ }^{\mathrm{a}}$ & $\checkmark$ & $\checkmark$ & & \\
\hline \multicolumn{5}{|l|}{ Past utilisation of services } \\
\hline Emergency services $^{b}$ & $\checkmark$ & & & \\
\hline Police or administrative interventions & $\checkmark$ & & & \\
\hline Community medical services $^{b}$ & $\checkmark$ & & $\checkmark$ & $\checkmark$ \\
\hline Outpatient services ${ }^{a, b}$ & $\checkmark$ & & $\checkmark$ & $\checkmark$ \\
\hline Social and welfare services ${ }^{b}$ & $\checkmark$ & & $\checkmark$ & $\checkmark$ \\
\hline Case management or outreach services & $\checkmark$ & $\checkmark$ & $\checkmark$ & $\checkmark$ \\
\hline Crisis planning ${ }^{b}$ & $\checkmark$ & $\checkmark$ & $\checkmark$ & $\checkmark$ \\
\hline Prescriptions & $\checkmark$ & $\checkmark$ & $\checkmark$ & $\checkmark$ \\
\hline \multicolumn{5}{|l|}{ Inpatient care during hospitalisation } \\
\hline Seclusion order & & $\checkmark$ & & \\
\hline Restraint order & & $\checkmark$ & & \\
\hline Electroconvulsive therapy ${ }^{b}$ & & $\checkmark$ & & \\
\hline Discharge plan or planning ${ }^{\mathrm{a}, \mathrm{b}}$ & & $\checkmark$ & & \\
\hline Therapies specific to hospitalisation $\mathrm{n}^{\mathrm{b}, \mathrm{d}}$ & & $\checkmark$ & & \\
\hline \multicolumn{5}{|l|}{ Outpatient care during follow-up } \\
\hline Outpatient service visit within 7 days of discharge from index admission & & & $\checkmark$ & \\
\hline Regular outpatient service visits & & & $\checkmark$ & $\checkmark$ \\
\hline Online medical examinations & & & $\checkmark$ & $\checkmark$ \\
\hline
\end{tabular}


Table 1 (continued)

\begin{tabular}{|c|c|c|c|c|}
\hline Item & $\begin{array}{l}\text { Time } \\
\text { of admission } \\
\text { (T1) }\end{array}$ & $\begin{array}{l}\text { Time } \\
\text { of discharge } \\
\text { (T2) }\end{array}$ & $\begin{array}{l}\text { 6-month } \\
\text { follow-up } \\
\text { (T3) }\end{array}$ & $\begin{array}{l}\text { 12-month } \\
\text { follow-up } \\
\text { (T4) }\end{array}$ \\
\hline \multicolumn{5}{|c|}{ Local social and welfare resources } \\
\hline \multicolumn{4}{|c|}{ Number of social and welfare service agencies in the patient's area of residence } & $\checkmark$ \\
\hline \multicolumn{5}{|c|}{ EQ-5D-5L EuroQol five dimensions and five levels, PSP Personal and Social Performance scale, SDS-J Sheehan Disability Scale-Japanese version } \\
\hline \multicolumn{5}{|c|}{ a Component of the READMIT index } \\
\hline \multicolumn{5}{|c|}{ b Possible responses are YES/NO } \\
\hline \multicolumn{5}{|c|}{$\begin{array}{l}\text { c Psychiatric present status include hallucinatory paranoid state, psychomotor excitement, stupor, Residual schizophrenia, depressive state, manic state, delirium, } \\
\text { twilight state and dementia symptom }\end{array}$} \\
\hline
\end{tabular}

corresponding to the primary aim of this study. The EQ-5D has been widely used in the area of mental health [42]. In addition, the scoring and language of the EQ$5 \mathrm{D}-5 \mathrm{~L}$ have been validated in multiple countries, including Japan [43-45]. The Sheehan Disability Scale (SDS), which is a self-reported disability measure, is used to assess participants' self-perceptions of problems in their lives. It consists of three items: work/academic activities, social activities, and family communication. Previous studies have confirmed that the Japanese version of the SDS has convergent validity with the Global Assessment of Functioning, good internal consistency, and extremely high test-retest reliability [46-48]. We will also employ a new scale to measure subjective views of empowerment and self-agency in people with mental illness. This scale asks participants whether they express themselves in their daily lives and whether they make decisions about the things that they encounter in their lives. The scale was tested in an assertive community treatment sample with relatively severe mental illness in Japan. Good factor validity has been confirmed [49]. Trained staff members help participants read and understand the sentences in each scale if necessary.

In addition to these repeated measures, the participants will complete an original questionnaire regarding their satisfaction with inpatient or outpatient care, subjective feelings about inpatient or outpatient care needs, and their relationships with family members at the time of discharge (T2) and 6-month follow-up (T3). Due to the paucity of brief, easily understandable, and scientifically validated scales designed specifically for assessing satisfaction with care and family relationships among people with mental illness in Japanese settings, we developed this original questionnaire through collaboration with service users who have experienced both inpatient and outpatient care (Additional file 1). We anticipate that participants will not be able to forthrightly answer these questions if staff members collect the questionnaire because the questions ask about the quality of care from staff members. Thus, the participants will complete the questionnaires without any help from staff members and mail them to the data center.

\section{Clinical, social, and other exposure and outcome measures}

At each assessment point, past hospitalizations (primary outcome), length of hospitalization, types of hospitalization (voluntary or involuntary), employment status, and social relationship status will be evaluated using medical records and participant interviews. In terms of other clinical variables, overall symptoms will be assessed based on previous studies conducted in the United States that evaluated the severity of symptoms using a 5-point grading scale $[6,7]$. In addition, the level of functioning will be assessed using the Japanese version of the Personal and Social Performance scale (PSP-J) [50]. The PSP has been used by clinicians worldwide as a rating tool for social and general functioning [51, 52]. The presence or absence of the following problematic behaviors will be abstracted from medical records: physical violence, self-harm, suicidal behavior, self-neglect, substance abuse, water intoxication, nuisance behavior, and nonadherence to treatment. Staff members will also make clinical judgements regarding the risk of the emergence of each type of problematic behavior during the ensuing 6 months. In addition to the actual presence of such behaviors, risk judgements will be determined because they can influence treatment planning. Past studies have suggested that judgements related to the risk of violence, based on readily accessible clinical information such as age, sex, and recent history of violence and drug use, may have predictive accuracy comparable to judgments based on more detailed risk assessment tools [53, 54]. Before the study started, staff members received a brief training 
session on rating each past problematic behavior and risk estimate.

In addition, study staff will collect data on assessment point-specific variables. At the time of admission (T1), information on sociodemographic characteristics (sex, age, living condition, pension, types of medical and social service utilization), diagnoses, existence of a crisis plan, and past prescriptions will be gathered. We will also assess current psychiatric statuses that are likely to affect self-reported measures at admission (T1), including hallucinatory paranoid state, psychomotor excitement, stupor, residual schizophrenia, depressive state, manic state, delirium, twilight state, and dementia symptoms. In addition, the READMIT items (e.g., comorbidities, past emergency service use; see Vigod et al.) will be included as the participants' demographic and background information [17]. At the time of discharge (T2), information on the patients' prescriptions and types of care provided during hospitalization will be collected. During follow-up assessments (T3 and T4), the outpatient services and prescriptions that participants received during the previous 6 months will be recorded. All information will primarily be gathered through medical records. If the medical records do not include an assessment item, trained staff members will ask the participants to provide additional data. In addition, we will collect data on the number of social or welfare service agencies in the patient's living area as an environmental geographical variable using the electronic Regional Mental Health Resources Analyzing Database (ReMHRAD, https://remhrad.ncnp.go.jp/), which can identify the number of service agencies in the patient's local government area of residence.

\section{Cost data}

Supplemental cost data will be collected from two participating hospitals to illustrate costs during the study period, although we will not use these data in the main analysis. Medical costs will be gathered using individual receipt data from the two hospitals. To gather the cost data of medical services used in medical institutions other than the two hospitals and social services, we will assess the number of such services used through the Japanese version of the Client Service Receipt Inventory (CSRI-J) [55]. The CRSI-J includes the cost of social security and other public or social allowances in addition to information about services. At each assessment point, staff members at the two hospitals will interview the participants about their service utilization using CSRI-J. The costs of services will be computed in reference to a previous Japanese cost study [56]. Specifically, medical service and social service costs will be calculated based on the unit costs in the National Health Insurance or the Services and Supports for Persons with Disabilities Act, respectively. In addition, the costs per hour of services from public sectors (e.g., city offices, local government, and public unemployment offices) will be estimated based on the average salaries earned by public officers of the average age in Japan.

\section{Sample size consideration}

Based on the primary aim of this study, the sample size was calculated using the effect size reported by a previous study. That study reported an association between readmission and QoL scores $(d=0.26)$ [20] and indicated the readmission rate in Japanese psychiatric hospitals (40\%, ratio of approximately 1 vs 1.5) [38]. In addition, the calculation was based on a power of $70 \%$ at a twosided significance level of $5 \%$. Based on these values, 430 participants will be needed. Assuming that $20 \%$ of the participants will not be discharged within 12 months and a $25 \%$ attrition rate [57], the final number of participants required was estimated to be 624 . This calculation was conducted using Stata version 15.

\section{Data analysis \\ Descriptive statistics}

Descriptive summary statistics will be calculated for sociodemographic characteristics, outcomes, and other exposures. These values will be presented as means, standard deviations, medians, interquartile ranges, frequencies, and proportions, as appropriate.

\section{Primary objectives}

For the primary objective, mixed-effects logistic regression will be performed to assess the relationship between the QoL score at discharge (T2) and readmission over a 12-month follow-up period beginning at discharge after the index admission. In the first model, we will adjust for sex, age, and diagnosis. Next, we will conduct an analysis that adjusts for the potential covariates suggested in previous studies, for example, function (PSP score), symptoms, accommodation, problematic behaviors, past hospitalization, comorbidities, and utilization of outreach services after discharge, in addition to sex, age, and diagnosis. Statistical significance will be set at 5\% (twotailed). In these models, we will only include participants with data for both the primary exposure (QoL score at discharge, T2) and the primary outcome (readmission over 12 months).

\section{Secondary objectives}

We will again conduct mixed-effects logistic regression to assess whether self-reported disability, empowerment/self-agency-related measure or service satisfaction with inpatient care at discharge (T2) affect readmission over a 12-month follow-up period beginning at 
discharge after the index admission, respectively. In addition, we will conduct an analysis that adjusts for the same potential covariates as the QoL analyses. We will only analyze patients with data on self-reported disability (T2), empowerment/self-agency-related measure (T2) or service satisfaction (T2) with inpatient care and readmission.

\section{Additional objectives}

We will also create mixed models with repeated measures to explore the impacts of readmission on changes in QoL, empowerment/self-agency-related measure, and self-reported disability. In this model, we will adjust for sex, age, diagnosis, baseline score at admission (T1) and present psychiatric status at admission (T1). In addition, we will explore other potential factors that influence the changes in patient-reported outcomes using mixed models with repeated measures. In this analysis, we will include at least the following variables: function (PSP score), length of hospital stay, living condition, and utilization of outreach services after discharge. Because the mixed-model method can accommodate missing data, we will use the data from patient-reported measures completed at least twice at the three follow-up assessments (T2-T4), in addition to the assessment at admission (T1).

\section{Discussion}

The present study will primarily examine the association between psychiatric readmission and patient-reported experiences, particularly QoL. Past studies have often focused on schizophrenia only or followed participants from the time of discharge. Apart from three German studies [11-16, 24, 30, 31, 58], few studies have followed participants who meet broad criteria from the time of admission to 12 months after discharge from the index admission to examine such an association. This study will provide new evidence regarding whether patients' subjective feelings about their lives influence clinical outcomes. In addition, this study will collect various types of data regarding factors potentially associated with readmission and patient-reported measures at the individual and service levels. If patient-reported experiences are able to predict future readmission after adjustment for potential covariates, then routine clinical practice should focus more on each patient's subjective perceptions of their illness and life. In addition, if improving patients' subjective feelings or experiences can prevent readmission, it may also contribute to reducing the cost of inpatient care. Conversely, if we identify a particular factor that influences the course of patient-reported outcomes (e.g., QoL) in community living after discharge, it will provide useful evidence for improving clinical and social services in the community setting. In addition, we will suggest that mental health and governmental institutions routinely assess such variables and accumulate data. Such findings might contribute to a future large-scale study using existing data as the value of large-scale observational studies in health research has dramatically increased in parallel with the development of national databases [59].

We recognize some study limitations. The first is related to the nature of observational data. The research findings may suggest key factors affecting readmission and patient-reported outcomes at 12 months of follow-up, but the study will not be able to clarify the effect of a particular intervention. Second, the generalizability of study findings will be limited, even though the study will be conducted at multiple sites across Japan. Participating institutions are limited to hospitals that are relatively accessible for research work in a Japanese context. The third limitation is the sampling procedure that will be used for this study, particularly for those who have very severe psychiatric symptoms at the time of admission (T1). The participants will consist only of patients who can voluntarily consent to study participation and can complete the self-rated questionnaire. In other words, the study will not be able to include patients who cannot respond to the questionnaire, for example, due to very severe symptoms. Although we will adjust for diagnoses and symptoms that are likely to affect participants' capacity to respond to patient-reported measures, the study will be affected by response bias. Measuring patients' views of themselves, their lives, and their satisfaction with treatment may be challenging in the acute psychiatric care setting, but these evaluations are not impossible, as shown by a previous study [24]. We specifically selected brief, self-rated measures for this study to help address this problem. However, another potential limitation is the EQ-5D-5L, which is used as a patient-reported experience and outcome measure and is the primary interest in this study. While the scale is a validated measure, it is not a gold standard for assessing QoL, particularly in patients with schizophrenia or bipolar disorder [42]. However, the scale has several advantages, such as its brevity, simplicity, utilization of economic evaluation, and the finding that its results can be compared among people with other disorders or among the general population [60].

Despite the potential methodological limitations, this study attempts to collect data on multiple variables. It may be difficult to gather data for patient-reported experiences and outcome measures repeatedly in a retrospective or a national database study. In addition, our protocol was developed through collaboration among researchers, clinicians and service users. The findings of this study will 
be valuable for understanding patients' views of hospitalization and community living.

\section{Dissemination of findings}

The study findings will be reported based on the Strengthening the Reporting of Observational Studies in Epidemiology (STROBE) Statement [61] in peer-reviewed publications and presented at relevant scientific conferences. In addition, the results will be summarized and submitted to the funding body (Ministry of Health, Labour, and Welfare of Japan) to fulfil grant-reporting requirements. We will also ask an organization involving mental health service users (Community Mental Health \& Welfare Bonding Organization, COMHBO) to help disseminate the study findings.

\section{Additional file}

Additional file 1. Questionnaire for service satisfaction and subjective family relationship.

\section{Abbreviations}

CSRI-J: Japanese version of the Client Service Receipt Inventory; EQ-5D-5L: EuroQOL Five Dimensions and Five Levels; PSP: Personal and Social Performance scale; QoL: quality of life; SDS: Sheehan Disability scale.

\section{Acknowledgements}

We are very grateful for the help and advice of Eriko Kobayashi, Keiko Miyazawa, Rie Sasaki, Takatsuna Sawada, Tomoyuki Uchinuno, Toshihiko Koseki with developing the questionnaire for this study. We wish to thank Dr. Yoshio Yamanouchi for assistance with recruiting the hospitals. We are also grateful to Ms. Aya Kumagami and Ms. Yasuko Miwa for their assistance with launching the study.

\section{Authors' contributions}

$\mathrm{SY}, \mathrm{YO}, \mathrm{JK}, \mathrm{AM}, \mathrm{MO}, \mathrm{HT}$, KY, and CF conceived this work. SY and CF secured the funding. All the authors contributed to the development and refinement of the study protocol. SY, YO, JK, AM, MO, AK, and CF drafted the manuscript. When we write the manuscripts based on the results of this study, we will not use a professional writer. All authors read and approved the final manuscript.

\section{Funding}

This work is supported by a Grant from the Japan Ministry of Health, Labour and Welfare, (H30-004: Study project for early discharge and community lives in psychiatric inpatients: cohort study). The study is also funded by another Grant from the Japan Ministry of Health, Labour and Welfare (H28-001: H28001: Policy research for improving the quality of community life of people with mental disorders). The funder has had no influence on developing the protocol or on future study processes, including data management, analysis, and interpretation of the results; however, the funder will play a role in monitoring whether the study proceeds according to the proposal plan. The contact information for the Japan Ministry of Health, Labor and Welfare is as follows: address, 1-2-2 Kasumigaseki Chiyoda Tokyo, 100-8916 Japan; Tel: 03-5253-1111

\section{Availability of data and materials}

The datasets generated and/or analyzed during the current study are not publicly available due to a relevant Japanese policy for Ethical Guidelines for Medicine for a person of interest and the ethical committee approval for this study but are available from the corresponding author on reasonable request.

\section{Ethics approval and consent to participate}

The ethical considerations of the current study, including the informed consent process and patient privacy measures, are based on the ethics guidelines for medical research in Japan [62]. The study protocol was approved by the Ethics Committee of the Graduate School of Medicine at Chiba University (no. 3154). If we amend our protocol, we will ask the Ethics Committee to revise it and will report this change in the paper.

\section{Consent for publication \\ Not applicable.}

\section{Competing interests}

The authors declare that they have no competing interests.

\section{Author details}

${ }^{1}$ Department of Community Mental Health \& Law, National Institute of Mental Health, National Center of Neurology and Psychiatry, 4-1-1 Ogawa-Higashi, Kodaira 187-8553, Japan. ${ }^{2}$ Translational Medical Center, National Center of Neurology and Psychiatry, 4-1-1 Ogawa-Higashi, Kodaira 187-8553, Japan. ${ }^{3}$ The Institute for Global Health Policy Research, Bureau of International Health Cooperation, National Center for Global Health and Medicine, 1-21-1 Toyama Shinjuku-ku, Tokyo 162-8655, Japan. ${ }^{4}$ Department of Psychiatry, Graduate School of Medicine, Chiba University, 1-8-1 Inohana, Chuo-ku, Chiba 260-8670, Japan. ${ }^{5}$ Department of Psychiatry, Gakuji-kai, Kimura Hospital, 6-19 Higashi-honcho, Chuo-ku, Chiba 260-8670, Japan. ${ }^{6}$ College of Education, Psychology and Human Studies, Aoyama Gakuin University, 4-4-25 Shibuya, Shibuya-ku, Tokyo 150-8366, Japan. ${ }^{7}$ Division of Medical Treatment and Rehabilitation, Center of Forensic Mental Health, Chiba University, 1-8-1 Inohana, Chuo-ku, Chiba 260-8670, Japan. ${ }^{8}$ Department of Psychiatry, Okayama Psychiatric Medical Center, 3-16 Shikata-Honmachi, Kita-ku, Okayama, Japan. ${ }^{9}$ Department of Human Care and Support, Toyo University, 48-1 Oka, Asaka 351-8510, Japan. ${ }^{10}$ Department of Psychiatry, Kitasato University School of Medicine, 1-15-1 Kitazato, Minami, Sagamihara 252-0374, Japan. ${ }^{11}$ Department of Psychiatry, Nara Medical University School of Medicine, 840 Shijo, Kashihara 634-8521, Japan.

Received: 30 March 2019 Accepted: 28 May 2019

Published online: 07 June 2019

\section{References}

1. Slade M, Amering M, Farkas M, Hamilton B, O'Hagan M, Panther G, et al. Uses and abuses of recovery: implementing recovery-oriented practices in mental health systems. World Psychiatry. 2014;13:12-20.

2. Anthony WA. Recovery from mental illness: the guiding vision of the mental health service system in the 1990s. Psychosoc Rehabil J. 1993:16:11-23

3. Winkler P, Barrett B, McCrone P, Csémy L, Janousková M, Höschl C. Deinstitutionalised patients, homelessness and imprisonment: systematic review. Br J Psychiatry. 2016;208:421-8.

4. Thornicroft G, Deb T, Henderson C. Community mental health care worldwide: current status and further developments. World Psychiatry. 2016;15:276-86.

5. Knapp M, Beecham J, McDaid D, Matosevic T, Smith M. The economic consequences of deinstitutionalisation of mental health services: lessons from a systematic review of European experience. Health Soc Care Community. 2010;19:113-25.

6. Strauss JS, Carpenter WT Jr. The prediction of outcome in schizophrenia: I. characteristics of outcome. Arch Gen Psychiatry. 1972;27:739-46.

7. Strauss JS, Carpenter WT Jr. The prediction of outcome in schizophrenia: II. relationships between predictor and outcome variables: a report from the who international pilot study of schizophrenia. Arch Gen Psychiatry. 1974;31:37-42

8. Stein LI, Test MA. Alternative to mental hospital treatment. I. Conceptual model, treatment program, and clinical evaluation. Arch Gen Psychiatry. 1980;37:392-7.

9. Thornicroft G, Gooch C, Dayson D. The TAPS project. 17: Readmission to hospital for long term psychiatric patients after discharge to the community. BMJ. 1992;305:996-8. 
10. Leff J, Trieman N, Knapp M, Hallam A. The TAPS Project: a report on 13 years of research, 1985-1998. Psychiatrist. 2000;24:165-8.

11. Donisi V, Tedeschi F, Wahlbeck K, Haaramo P, Amaddeo F. Pre-discharge factors predicting readmissions of psychiatric patients: a systematic review of the literature. BMC Psychiatry. 2016;16:449.

12. Kalseth J, Lassemo E, Wahlbeck K, Haaramo P, Magnussen J. Psychiatric readmissions and their association with environmental and health system characteristics: a systematic review of the literature. BMC Psychiatry. 2016:16:376.

13. Sfetcu R, Musat S, Haaramo P, Ciutan M, Scintee G, Vladescu C, et al. Overview of post-discharge predictors for psychiatric re-hospitalisations: a systematic review of the literature. BMC Psychiatry. 2017;17:227.

14. Vigod SN, Kurdyak PA, Dennis C-L, Leszcz T, Taylor VH, Blumberger DM, et al. Transitional interventions to reduce early psychiatric readmissions in adults: systematic review. Br J Psychiatry. 2013;202:187-94.

15. Šprah $L$, Dernovšek $M Z$, Wahlbeck $K$, Haaramo P. Psychiatric readmissions and their association with physical comorbidity: a systematic literature review. BMC Psychiatry. 2017;17:2.

16. Lafeuille M-H, Dean J, Carter V, Duh MS, Fastenau J, Dirani R, et al. Systematic review of long-acting injectables versus oral atypical antipsychotics on hospitalization in schizophrenia. Curr Med Res Opin. 2014;30:1643-55.

17. Vigod SN, Kurdyak PA, Seitz D, Herrmann N, Fung K, Lin E, et al. READMIT: a clinical risk index to predict 30-day readmission after discharge from acute psychiatric units. J Psychiatr Res. 2015;61:205-13.

18. Eack SM, Newhill CE. Psychiatric symptoms and quality of life in schizophrenia: a meta-analysis. Schizophr Bull. 2007;33:1225-37.

19. Van Eck RM, Burger TJ, Vellinga A, Schirmbeck F, de Haan L. The relationship between clinical and personal recovery in patients with schizophrenia spectrum disorders: a systematic review and meta-analysis. Schizophr Bull. 2018;44:631-42.

20. Shadmi E, Gelkopf M, Garber-Epstein P, Baloush-Kleinman V, Doudai R, Roe D. Routine patient reported outcomes as predictors of psychiatric rehospitalization. Schizophr Res. 2018;192:119-23.

21. Niimura J, Tanoue M, Nakanishi M. Challenges following discharge from acute psychiatric inpatient care in Japan: patients' perspectives. J Psychiatr Ment Health Nurs. 2016;23:576-84.

22. Newton-Howes G, Mullen R. Coercion in psychiatric care: systematic review of correlates and themes. Psychiatr Serv. 2011;62:465-70.

23. Dixon LB, Holoshitz Y, Nossel I. Treatment engagement of individuals experiencing mental illness: review and update. World Psychiatry. 2016;15:13-20.

24. Priebe S, Katsakou C, Amos T, Leese M, Morriss R, Rose D, et al. Patients' views and readmissions 1 year after involuntary hospitalisation. $\mathrm{Br} J$ Psychiatry. 2009;194:49-54.

25. Thornicroft G, Slade M. New trends in assessing the outcomes of mental health interventions. World Psychiatry. 2014;13:118-24.

26. Dwinger $\mathrm{S}$, Dirmaier J, Herbarth L, König HH, Eckardt M, Kriston L, et al. Telephone-based health coaching for chronically ill patients: study protocol for a randomized controlled trial. Trials. 2013;14:337.

27. Reininghaus $U$, Priebe S. Measuring patient-reported outcomes in psychosis: conceptual and methodological review. Br J Psychiatry. 2012;201:262-7.

28. Kendrick T, El-Gohary M, Stuart B, Gilbody S, Churchill R, Aiken L, et al. Routine use of patient reported outcome measures (PROMs) for improving treatment of common mental health disorders in adults. Cochrane Database Syst Rev. 2016;7:CD011119.

29. Slade M. 100 ways to support recovery: a guide for mental health professionals. London: Rethink; 2009

30. Priebe S, Roeder-Wanner UU, Kaiser W. Quality of life in first-admitted schizophrenia patients: a follow-up study. Psychol Med. 2000;30:225-30.

31. Lay B, Drack T, Bleiker M, Lengler S, Blank C, Rossler W. Preventing compulsory admission to psychiatric inpatient care: perceived coercion, empowerment, and self-reported mental health functioning after 12 months of preventive monitoring. Front Psychiatry. 2015;6:161.

32. Kingston T, Scully PJ, Browne DJ, Baldwin PA, Kinsella A, O'Callaghan E, et al. Functional outcome and service engagement in major depressive disorder with psychotic features: comparisons with schizophrenia, schizoaffective disorder and bipolar disorder in a 6-year follow-up of the Cavan-Monaghan First Episode Psychosis Study (CAMFEPS). CNS Neurosci Ther. 2018;24:633-40.
33. Fowler JC, Madan A, Allen JG, Ellis T, Mahoney J, Hardesty S, et al. Improvement in health-related quality of life among adults with serious mental illness receiving inpatient treatment: a prospective cohort study. J Clin Psychiatry. 2015;76:e632-8.

34. Priebe S, Kaiser W, Huxley PJ, Roder-Wanner U-U, Rudolf H. Do different subjective evaluation criteria reflect distinct constructs? J Nerv Ment Dis. 1998;186:385-92.

35. Schöttle D, Schimmelmann BG, Ruppelt F, Bussopulos A, Frieling M, Nika $E$, et al. Effectiveness of integrated care including therapeutic assertive community treatment in severe schizophrenia-spectrum and bipolar I disorders: four-year follow-up of the ACCESS II study. PLoS ONE. 2018;13:e0192929.

36. WHO. Mental health atlas 2014. Geneva: World Health Organization; 2014

37. Kasai K, Ando S, Kanehara A, Kumakura Y, Kondo S, Fukuda M, et al. Strengthening community mental health services in Japan. Lancet Psychiatry. 2017:4:268-70.

38. Department of Mental Health Policy. Annual data of mental health and welfare Kodaira: National Center of Neurology and Psychiatry; 2018. https ://www.ncnp.go.jp/nimh/seisaku/data/.

39. Okumura Y, Sugiyama N, Noda T, Tachimori H. Psychiatric admissions and length of stay during fiscal years 2014 and 2015 in Japan: a retrospective cohort study using a nationwide claims database. J Epidemiol. 2018. https://doi.org/10.2188/jea.je20180096.

40. Okumura Y, Sugiyama N, Noda T, Sakata N. Association of high psychiatrist staffing with prolonged hospitalization, follow-up visits, and readmission in acute psychiatric units: a retrospective cohort study using a nationwide claims database. Neuropsychiatr Dis Treat. 2018;14:893-902.

41. Okumura Y, Sugiyama N, Noda T. Timely follow-up visits after psychiatric hospitalization and readmission in schizophrenia and bipolar disorder in Japan. Psychiatry Res. 2018;270:490-5.

42. Brazier J, Connell J, Papaioannou D, Mukuria C, Mulhern B, Peasgood T, et al. A systematic review, psychometric analysis and qualitative assessment of generic preference-based measures of health in mental health populations and the estimation of mapping functions from widely used specific measures. Health Technol Assess. 2014;18:vii-viii, xiii-xxv, 1-188.

43. Herdman M, Gudex C, Lloyd A, Janssen M, Kind P, Parkin D, et al. Development and preliminary testing of the new five-level version of EQ-5D (EQ-5D-5L). Qual Life Res. 2011:20:1727-36.

44. van Hout B, Janssen MF, Feng YS, Kohlmann T, Busschbach J, Golicki D, et al. Interim scoring for the EQ-5D-5L: mapping the EQ-5D-5L to EQ5D-3L value sets. Value Health. 2012;15:708-15.

45. Ikeda S, Shiroiwa T, Igarashi A, Noto S, Fukuda T, Saito S, et al. Developing a Japanese version of the EQ-5D-5L value set. J Natl Inst Public Health. 2015:64:47-55

46. Yoshida T, Otsubo T, Tsuchida H, Wada Y, Kamijima K, Fukui K. Reliability and validity of the Sheehan Disability Scale-Japanese version. Jpn J Clin Psychopharmacol. 2004;7:1645-53.

47. Sheehan D, Harnett-Sheehan K, Raj B. The measurement of disability. Int Clin Psychopharmacol. 1996;11:89-95.

48. Sheehan $\mathrm{KH}$, Sheehan DV. Assessing treatment effects in clinical trials with the Discan metric of the Sheehan Disability Scale. Int Clin Psychopharmacol. 2008;23:70-83.

49. Yamaguchi S, Shiozawa T, Matsunaga A, Utako S, Ayano T, Chiyo F. Development and psychometric properties of a new measure of self-agency and empowerment for people with mental illness. (in preparation). 2019.

50. Inada S. Japanese version of Personal and Social Performance Scale: A training sheet for evaluation version 1.0. Tokyo: Japanese Society of Psychiatric Rating Scales; 2011.

51. Alonso J, Angermeyer M, Bernert S, Bruffaerts R, Brugha T, Bryson H, et al. Use of mental health services in Europe: results from the European Study of the Epidemiology of Mental Disorders (ESEMeD) project. Acta Psychiatr Scand. 2004;109:47-54.

52. Burgess PM, Harris MG, Coombs T, Pirkis JE. A systematic review of clinician-rated instruments to assess adults' levels of functioning in specialised public sector mental health services. Aust N Z J Psychiatry. 2017:51:338-54.

53. Wootton L, Buchanan A, Leese M, Tyrer P, Burns T, Creed F, et al. Violence in psychosis: estimating the predictive validity of readily accessible clinical information in a community sample. Schizophr Res. 2008;101:176-84. 
54. O'Shea LE, Dickens GL. Role of assessment components and recent adverse outcomes in risk estimation and prediction: use of the Short Term Assessment of Risk and Treatability (START) in an adult secure inpatient mental health service. Psychiatry Res. 2016;240:398-405.

55. Yamaguchi S, Shimodaira M, Yoshida K, Sato S, Takahara Y, Maeda K, et al. An instrument for economic evaluation of mental health services: development of the Client Service Receipt Inventory-Japanese version. Seishin Igaku. 2012;54:1225-36.

56. Yamaguchi S, Sato S, Horio N, Yoshida K, Shimodaira M, Taneda A, et al. Cost-effectiveness of cognitive remediation and supported employment for people with mental illness: a randomized controlled trial. Psychol Med. 2017:47:53-65.

57. Yamaguchi S, Furuie M, Yoshida K, Sato S, Shimodaira M, Taneda A, et al. Utilization and costs of community services for people with severe mental illness after discharge from hospitals: a nested cross-sectional survey. Jpn J Psychiatr Rehabil. 2015;19:52-62.

58. Lay B, Blank C, Lengler S, DrackT, Bleiker M, Rossler W. Preventing compulsory admission to psychiatric inpatient care using psycho-education and monitoring: feasibility and outcomes after 12 months. Eur Arch Psychiatry Clin Neurosci. 2015:265:209-17.
59. Booth CM, Tannock IF. Randomised controlled trials and populationbased observational research: partners in the evolution of medical evidence. Br J Cancer. 2014;110:551.

60. Brazier J. Is the EQ-5D fit for purpose in mental health? Br J Psychiatry 2010;197:348-9.

61. von Elm E, Altman DG, Egger M, Pocock SJ, Gotzsche PC, Vandenbroucke JP, et al. Strengthening the Reporting of Observational Studies in Epidemiology (STROBE) statement: guidelines for reporting observational studies. BMJ. 2007:335:806-8.

62. MEXT, MHLW. Outline of ethical guidelines for medical and health research involving human subjects: version 20170529. Tokyo: Ministry of Education, Culture, Sports, Science and Technology and Ministry of Health, Labour and Welfare; 2017

\section{Publisher's Note}

Springer Nature remains neutral with regard to jurisdictional claims in published maps and institutional affiliations.
Ready to submit your research? Choose BMC and benefit from:

- fast, convenient online submission

- thorough peer review by experienced researchers in your field

- rapid publication on acceptance

- support for research data, including large and complex data types

- gold Open Access which fosters wider collaboration and increased citations

- maximum visibility for your research: over $100 \mathrm{M}$ website views per year

At BMC, research is always in progress.

Learn more biomedcentral.com/submissions 